\title{
ASYMPTOTICALLY MINIMAX ESTIMATION OF ORDER-CONSTRAINED PARAMETERS AND EIGENFUNCTIONS OF THE LAPLACIAN ON THE BALL
}

\author{
Adam KORÁNYI ${ }^{\mathrm{a}}$, K. Brenda MACGIBBON ${ }^{\mathrm{b}}$

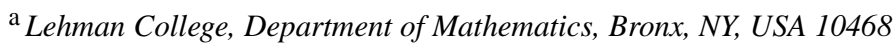 \\ ${ }^{\mathrm{b}}$ GERAD and Université du Québec à Montréal, Département de mathématiques, \\ C.P. 8888, Succursale Centre-ville, Montréal, QC, Canada H3C $3 P 8$
}

Received 3 January 2000, revised 8 June 2001

ABSTRACT. - Inspired by previous results on asymptotic minimax estimation for a ball of increasing radius in $\mathbb{R}^{n}$, we study the analogous problem for domains of importance in orderrestricted inference. In particular, we study domains that are formed by the intersection of a ball and a fundamental chamber of a finite reflection group in $\mathbb{R}^{n}$. We show (1) how to obtain the principal eigenfunction of such a domain and asymptotically, the related least favourable distribution for the associated minimax problem, (2) that the order and positivity constraints in the usual statistical problems generate such chambers and (3) and in an analogous way to the work of Bickel [5] in the one dimensional case, how to find the asymptotic minimax risk and the second order asymptotic minimax estimate for such a domain. @ 2002 Éditions scientifiques et médicales Elsevier SAS

AMS classification: Primary 62G05; 62C20; secondary 33C10; 31B20

Keywords: Asymptotic risk; Bayes; Least favorable prior distribution; Reflection groups; Boundary value problems

RÉSUMÉ. - Inspiré par les résultats existants sur l'estimation asymptotique minimax pour une boule de rayon croissant dans $\mathbb{R}^{n}$, nous étudions le problème analogue pour certains domaines importants en inférence avec des contraintes d'ordre. Plus particulièrement, nous étudions les domaines formés par l'intersection d'une boule et d'une chambre fondamentale d'un groupe de réflexions dans $\mathbb{R}^{n}$. Nous montrons (1) comment obtenir la fonction propre principale d'un tel domaine et la loi a priori la moins favorable asymptotiquement pour le problème du minimax associé, (2) que les contraintes d'ordre et de positivité dans les problèmes statistiques usuels engendrent de telles chambres et (3) d'une manière analogue au travail de Bickel [5] dans le cas unidimensionnel, comment trouver le risque asymptotiquement minimax et l'estimateur asymptotiquement minimax du second ordre sur un tel domaine. (๖ 2002 Éditions scientifiques et médicales Elsevier SAS

E-mail addresses: adam@alpha.lehman.cuny.edu (A. Korányi), macgibbon.brenda@uqam.ca (K.B. MacGibbon). 


\section{Introduction}

In many parametric statistical estimation problems, there is definite prior information concerning the values of a parameter vector $(\omega)$. There may be bounds on the individual components $(\omega)_{i}$ such as "the norm of $\omega$ is at most $c$ " or "a subset of the $\omega_{i}$ are non-negative" or the " $\omega_{i}$ 's are non-increasing in $i$ ". Many computationally feasible estimation methods have been developed to capitalize on such information. How could one theoretically compare the performance of various possible estimators when such prior information is present? One common, admittedly conservative, approach is the worst-case analysis: given some error measure, compute the maximum expected error over the restricted parameter space, and then seek the estimator that minimizes this maximum risk. The resulting best or minimax risk provides a benchmark against which to measure other estimators.

Here we recall various definitions of statistical decision theory associated with the estimation of an unknown parameter vector $\omega$ given an observation of $x \in \mathbf{X}$, where $\mathbf{X}$ is a random vector whose distribution depends on the parameter $\omega \in \Omega$, the parameter space. A solution consists of a nonrandomized estimator or decision procedure $\delta$, which is a measurable function from the sample space $\mathbf{X} \rightarrow \boldsymbol{\Omega}$. Let $\mathcal{A}$ denote the space of all possible estimates. A risk function $R(\delta, \omega)$ characterizes the performance of a decision procedure $\delta$ for each value of the parameter $\omega$. The risk function is usually defined in terms of an underlying loss function $L(\delta, \omega)$ which maps $\mathcal{A} \times \Omega \rightarrow \mathbb{R}^{+} \cup\{0\}$ (where $\mathbb{R}^{+}$is the positive real line). To be able to confine our attention to nonrandomized estimators, it will be assumed that the loss function is convex in $\delta$ and that $\mathcal{A}$ and $\Omega$ are also convex. The loss function will usually be assumed to equal the quadratic loss function $\sum\left(\delta_{i}-\omega_{i}\right)^{2}$. The risk of an estimator $\delta$ when $\omega$ is true, $R(\delta, \omega)$, is then the average loss incurred from using $\delta$; that is, $R(\delta, \omega)=E_{\omega} L(\delta(X), \omega)$. An estimator $\delta^{*}$ is minimax for the above problem, if

$$
\sup _{\omega \in \Omega} R\left(\delta^{*}, \omega\right) \leqslant \sup _{\omega \in \Omega} R(\delta, \omega)
$$

for all $\delta \in \mathcal{A}$. We will let $\rho(\Omega)$ denote the minimax risk on $\Omega$; i.e., $\rho(\Omega)=$ $\inf _{\delta \in \mathcal{A}} \sup _{\omega \in \Omega} R(\omega, \delta)$. Note that if $\Omega=\mathbb{R}^{n}$ then for the normal mean estimation problem $\rho\left(\mathbb{R}^{n}\right)=n$.

Minimax problems are often solved by considering the corresponding Bayes problems. A distribution or prior probability measure $\pi$ is specified on the parameter space $\Omega$, and a measure of the performance of a procedure $\delta$ is given by its Bayes risk

$$
r(\delta, \pi)=\int_{\Omega} R(\delta, \omega) \pi(\mathrm{d} \omega) .
$$

Now $\delta_{\pi}$ is called the Bayes procedure with respect to the prior probability measure $\pi$ if $\delta_{\pi}$ minimizes the Bayes risk. The Bayes risk $r(\pi)$ of a distribution or prior probability measure $\pi$ on $\Omega$ is defined as $r(\pi)=r\left(\delta_{\pi}, \pi\right)$. A distribution or prior probability measure $\pi^{*}$ is "least favorable" if its Bayes risk is greater than or equal to that of any other distribution. Subject to the decision problem satisfying sufficient regularity conditions, a least favorable prior distribution exists and the corresponding Bayes procedure is minimax (see Wald [29], Ferguson [12], Brown [7], and Kempthorne [18]). 
Here we consider the normal mean estimation problem; that is, $X$ is a $N_{n}\left(\omega, I_{n}\right)$ random vector where $n$ represents the dimension of the space, $I_{n}$ the $(n \times n)$ identity matrix and let us assume that the vector $\omega$ is contained in the parameter space $\Omega$ which is a closed bounded convex domain in $\mathbb{R}^{n}$.

Casella and Strawderman [9] first found exact minimax estimates for intervals of the form [ $-t, t]$ for small $t$ for this problem in one dimension. (See also Zinzius [31].) Donoho, Liu and MacGibbon [10] studied minimax estimates and affine-minimax estimates for the problem of estimating the mean of a standard Gaussian shift when the mean is known to lie in an orthosymmetric, convex and quadratically convex set in $\ell_{2}$. However, for many closed bounded domains $\Omega$ of $\mathbb{R}^{n}$ (satisfying $\Omega=\overline{\operatorname{int} \Omega}$ ), the finding of exact minimax solutions is analytically intractable. A more soluble analytical problem involves the study of asymptotically minimax estimates; that is, we can consider the asymptotic behaviour of the minimax risk

$$
\rho(t \Omega)=\inf _{\delta} \sup _{\omega \in t \Omega} E_{\omega} L(\delta(X), \omega)
$$

for positive $t$ and the construction of such asymptotically minimax estimators as $t \rightarrow \infty$.

The connection between asymptotic minimax risk and the principal eigenvalue of an elliptic equation was first elaborated in a series of papers by Levit [19-21] and Berkin and Levit [4] which studied asymptotic second-order minimax estimators under a general class of loss functions in Gaussian and locally asymptotic Gaussian settings, and connected this problem with the principal eigenvalue of the Laplace (or more generally, second order uniformly elliptic) equation in the domain in which the parameter lies. Bickel [5] independently derived the results for intervals and balls in the Gaussian setting for squared error loss, obtaining explicit second-order asymptotically minimax estimates by suitably rescaling eigenfunctions of the sphere. Johnstone and MacGibbon [16,17] related the problem of finding asymptotic minimax estimates of a bounded Poisson vector to the Gaussian one.

The solution to the Dirichlet problem is easily seen to be related to asymptotically minimax estimation of a normal mean vector (see, e.g., Berkin and Levit [4], Levit [1921], Johnstone and MacGibbon [17]). Essentially, finding a minimax estimator with respect to a density $f$ on $\Omega$ is equivalent to minimizing the Fisher information $I(f)=$ $\int f^{-1}|\nabla f|^{2}$. Since, Fisher information $I$ and the energy functional $I^{*}$ in the classical Dirichlet problem are related by $I^{*}(v)=I\left(v^{2}\right) / 4$ the problem of minimax estimation becomes a search for principal eigenfunctions of the laplacian.

More precisely, using the solution to the classical Dirichlet problem for a bounded domain (not too irregular) $\Omega$ in $\mathbb{R}^{n}$ (see, e.g., Gilbarg and Trudinger [14]), we have the following general theorem (for a discussion of the proof and applications, see, e.g., Berkin and Levit [4], Johnstone and MacGibbon [16,17]).

THEOREM 1.1. - For $\Omega$ as above and t positive,

(i) The minimax risk for the n-dimensional problem just described is

$$
\rho(t \Omega)=\inf _{\delta \in D} \sup _{\omega \in t \Omega} E_{\omega} \sum_{i=1}^{n}\left[\delta_{i}(X)-\omega_{i}\right]^{2}=n-4 t^{-2} \lambda(\Omega)+\mathrm{o}\left(t^{-2}\right),
$$


where $\lambda(\Omega)$ denotes the minimum eigenvalue of the Laplace operator $\Delta=\sum_{1}^{n} \partial^{2} / \partial \omega_{j}^{2}$ on $\Omega$, i.e., the smallest $\lambda$ for which the equation

$$
\begin{array}{ll}
\Delta v(\omega)=-\lambda v(\omega), & \omega \in \operatorname{int} \Omega, \\
v=0, & \omega \in \partial \Omega
\end{array}
$$

has a non-zero solution. The eigenspace corresponding to $\lambda(\Omega)$ is one-dimensional, and the corresponding eigenfunction $v_{\Omega}(\omega)=v(\omega, \Omega)\left(\right.$ or $\left.-v_{\Omega}(\omega)\right)$ is strictly positive on $\Omega$. Assume that $v_{\Omega}$ is normalised so that $\int_{\Omega} v_{\Omega}^{2}=1$.

(ii) Let $G_{t}^{*}(\mathrm{~d} \omega)$ denote a least favorable prior distribution for the region $\Omega_{t}=$ $t \Omega(t>0)$ and $G_{1, \Omega}^{* t}(\mathrm{~d} \omega)$ the corresponding prior rescaled to $\Omega$. If we let $g_{1}(\omega)=v_{\Omega}^{2}(\omega)$ for $\omega \in \Omega$ and $g_{1}(\omega)=0$ for $\omega \notin \Omega$, then the probability measure $G_{1}(\mathrm{~d} \omega)=g_{1}(\omega) \mathrm{d} \omega$ is the weak limit of the (rescaled) least favorable distributions $G_{1, \Omega}^{* t}$. If $G_{t}(\mathrm{~d} \omega)$ represents the distribution corresponding to $G_{1}(\mathrm{~d} \omega)$ scaled up to $t \Omega$, that is, whose density is given by $g_{t}(x)=t^{-n} g_{\Omega}\left(x t^{-1}\right)$ where $g_{\Omega}(\omega)=g_{1}(\omega)$, then the $G_{t}$ are approximately least favorable in the following sense:

$$
\rho(t)=r\left(G_{t}\right)+\mathrm{o}\left(t^{-2}\right) .
$$

The proof depends on an easily proved identity, called Brown's identity (see Brown [7], Bickel [5], Brown and Gajek [8]) which says that for any prior distribution $G$ one has

$$
\delta_{G}(x)=x+\operatorname{grad} \log \left(g * \phi_{n}\right),
$$

where $g$ is the density of $G$ (which is set to 0 outside of its domain of definition $\Omega)$ and $\phi_{n}$ represents the standard normal $n$-dimensional density function and $r(G)=$ $n-I\left(g * \phi_{n}\right)$, where $I\left(g * \phi_{n}\right)$ is the Fisher information.

So far, the only known explicit solutions for the asymptotically minimax estimation of a bounded Gaussian vector were obtained on the sphere, an orthosymmetric domain, or on rectangles, which are products of orthosymmetric domains. However, much interesting statistical research has also occurred in parameter estimation under positivity or order constraints (for an overview of this subject, see Robertson, Wright, and Dykstra [25]). For example, the estimation of a Gaussian vector under order constraints on the components has been shown to be a necessary part of the solution to some statistical problems in toxicology experiments (Schoenfeld [26]).

The prior information about $\omega$ for such a problem could consist of it being contained in some domain $\Omega \subset \mathbb{R}^{n}$ such as the domain given by the inequalities $\omega_{1} \geqslant \omega_{2} \geqslant \cdots \geqslant \omega_{n}$ or $\omega_{1} \geqslant \omega_{2} \geqslant \cdots \geqslant \omega_{n} \geqslant 0$. Such inequalities arise in many situations in order restricted inference (Robertson et al. [25]). These two domains are well known as the standard fundamental domains (chambers) for certain finite groups generated by reflections (the groups of permutations, respectively, signed permutations of the coordinates). So, with the same effort, we may ask the question about the fundamental chamber $C$ of an arbitrary finite reflection group $W$. We cannot handle these domains directly, one reason being that Theorem 1.1 works smoothly only for bounded domains $\Omega$. But what can be done, and is still useful, is to consider $\Omega=C \cap B$ where $B$ is the unit ball of $\mathbb{R}^{n}$. Using a 
symmetry argument used before by Berard [1] and Berard and Besson [2] in the case of the intersection of the sphere with a Weyl chamber (see also Urakawa [27,28]), we show how to obtain from the class of eigenfunctions on the ball the principal eigenfunctions of $\Omega$. Then the asymptotically least favourable distributions can be derived and second order asymptotically minimax estimates for the associated minimax problem on $t \Omega$ can also be constructed in a manner analogous to Bickel's work [5] in the one dimensional case.

\section{Asymptotically least favorable prior distributions on a bounded domain equal to the intersection of a ball and the fundamental chamber of a reflection group}

Here our goal is to study some of the important domains for order restricted statistical inference and to obtain asymptotically least favorable prior distributions for such domains. The four chosen, each of which is a subset of the unit ball $B$ in $\mathbb{R}^{n}$, are as follows:

$$
\begin{aligned}
& \mathcal{S}=\left\{x \in \mathbb{R}^{n}:|x| \leqslant 1, x_{1} \geqslant 0\right\}, \\
& \mathcal{Q}=\left\{x \in \mathbb{R}^{n}:|x| \leqslant 1 \text { and } x_{1}, \ldots, x_{n} \geqslant 0\right\} \quad \text { and } \\
& \mathcal{A}=\left\{x \in \mathbb{R}^{n}:|x| \leqslant 1 \text { and } x_{1} \geqslant x_{2} \geqslant \cdots \geqslant x_{n}\right\}, \\
& \mathcal{B}=\left\{x \in \mathbb{R}^{n}:|x| \leqslant 1 \text { and } x_{1} \geqslant x_{2} \geqslant \cdots \geqslant x_{n} \geqslant 0\right\} .
\end{aligned}
$$

In order to achieve this, we first study the eigenfunctions on the unit ball; then a general theorem about the eigenfunctions of a domain equal to the intersection of a ball and the fundamental chamber of a reflection group is proved. The results for the domains given in (4) and for other interesting domains of this type then follow as special cases.

In the following, we use the notation $j_{v}(r)=r^{-v} J_{v}(r)$, where $J_{v}$ is the standard Bessel function with index $v$. We denote by $k_{v, 1}<k_{v, 2}<\cdots$ the positive zeroes of $J_{v}$ (hence also of $j_{v}$ ). We recall that $j_{v}(r)$ is an entire analytic function of $r$, for every $v$ (Bateman II, p. 4 [11]).

In order to obtain asymptotically minimax estimates for $t \Omega$ where $\Omega=C \cap B$, we must have an expression for the principal eigenfunction $h$ and the corresponding eigenvalue $\lambda$ for $\Omega$. The key idea of the proof is a symmetry argument also used in a similar context for the sphere by Berard [1] and Berard and Besson [2] (see also Urakawa $[27,28])$. It can be summarized as follows.

Let $W$ denote a finite group generated by reflections of $\mathbb{R}^{n}$. Then we have a set of hyperplanes $\mathcal{Z}$, invariant under $W$, such that the orthogonal reflections $s_{H}$ with $H \in \mathcal{Z}$ generate $W$. The connected components of $\mathbb{R}^{n} \backslash \bigcup_{H \in \mathcal{Z}} H$, the complement of all the $H \in \mathcal{Z}$, are called fundamental domains or chambers for $W$.

Each of the bounded domains $\mathcal{S}, \mathcal{Q}, \mathcal{A}, \mathcal{B}$ arises in this way as the intersection of the ball $B$ with a fundamental chamber $C$ of a certain $W$. Now the eigenfunctions of the Dirichlet problem for the ball $B$ can all be written as products of Bessel functions and harmonic polynomials. Then one can prove that the $W$-anti-invariant elements of $L^{2}(B)$ restricted to $C$ give exactly $L^{2}(C \cap B)$. Using some known facts about zeroes of Bessel 
functions the problem now reduces to finding the anti-invariant harmonic polynomial of least degree.

THEOREM 2.1. - Up to an arbitrary constant factor the solutions of the eigenvalue problem for the $n$ dimensional ball with Dirichlet boundary condition are exactly:

$$
f(x)=j_{v}\left(|x| k_{v, m}\right) h_{l}(x)
$$

where $v=l+\frac{n-2}{2}, l=0,1,2, \ldots, m=1,2, \ldots$, where $h_{l} \in \mathcal{H}_{l}$ is a homogeneous harmonic polynomial of degree $l$, and where $k_{v, m}$ is the mth zero of $J_{v}$. The corresponding eigenvalue is $k_{v, m}^{2}$.

The principal eigenfunction is given by $l=0, m=1$, that is:

$$
j_{\frac{n-2}{2}}\left(|x| k_{\frac{n-2}{2}, 1}\right)
$$

Proof. - For the proof of (5) see Theorem 2.66, p. 107 of Folland [13].

The principal eigenfunction is the one given by $l=0, m=1$ as in (6) because $k_{v, 1}<$ $k_{v+1,1}<k_{v, 2}<k_{v+1,2}<\cdots$ (see Watson, [30] p. 479 or Bateman II, [11] p. 59).

Now let us consider the general case. Henceforth $W$ will denote a finite group generated by reflections in $\mathbb{R}^{n}, C$ a fundamental chamber for $W$. More precisely, let $\mathcal{Z}$ be a finite set of hyperplanes in $\mathbb{R}^{n}$, such that the orthogonal reflection $s_{H}$ with respect to each $H \in \mathcal{Z}$ preserves the set $\mathcal{Z}$, and let $W$ be the group generated by all the $s_{H}(H \in \mathcal{Z}) . W$ is a finite Coxeter group; canonically associated with $W$ are natural numbers $m_{1}, \ldots, m_{n}$ called the exponents of $W$ (cf. Bourbaki, [6] Ch. 5, $\S 6$, No 2). The connected components of $\mathbb{R}^{n} \backslash \bigcup_{H \in \mathcal{Z}} H$ are called chambers. It is well known (Bourbaki, [6] Ch. 5, $\S 3$, Thm. 1 (p. 74)) that $W$ is simply transitive on the set of chambers. Let us fix one of them and call it $C$.

We choose a unit vector $e_{H}$ orthogonal to $H$ for each $H$ so that $\left(x \mid e_{H}\right)>0, \forall x \in C$. We note that,

$$
\Delta(f \circ w)=(\Delta f) \circ w
$$

for all $w \in W$ and all functions $f$, where $\Delta$ denotes the laplacian. This is clear since each $w$ is orthogonal (since all $s_{H}$ are orthogonal). In particular, if $f$ is harmonic, so is $f \circ w$. We say that a function $f$ is invariant if $f \circ w=f(\forall w \in W)$ and anti-invariant if $f \circ w=(\operatorname{det} w) f(\forall w \in W)$.

Let $\Pi$ be defined, $\forall x \in \mathbb{R}^{n}$, by:

$$
\Pi(x)=\prod_{H \in \mathcal{Z}}\left(x \mid e_{H}\right)
$$

THEOREM 2.2. - Let $W, C$ and $\Pi$ be defined as above and let $\Omega=B \cap C$, where $B$ is the unit ball in $\mathbb{R}^{n}$. Then the least favorable prior density on $\Omega$ is given by

$$
g_{\Omega}(x)=c_{\Omega} j_{v}\left(|x| k_{v, 1}\right)^{2}(\Pi(x))^{2}, \quad x \in \Omega
$$


with $v=N+\frac{n-2}{2}$ (where $N=|\mathcal{Z}|$, the number of planes in $\mathcal{Z}$ and also equals the degree of $\Pi$ ) and

$$
c_{\Omega}=2^{2 N} \pi^{-n / 2} \Gamma(v+1)\left(J_{v+1}\left(k_{v, 1}\right)\right)^{-2}\left(\prod_{l=1}^{n}\left(m_{i} !\right)\right)^{-1}
$$

where $m, \ldots, m_{n}$ are the exponents of $W$.

Proof. - The first part of the proof, the determination of the eigenfunctions of $\Omega$ can be found in Berard [1] and Berard and Besson [2] together with Urakawa [27,28]. For completeness we include it here.

We define the operator Alt on functions by:

$$
(\operatorname{Alt} f)(x)=\frac{1}{|W|} \sum_{w \in W}(\operatorname{det} w) f(w x),
$$

where $|W|=$ \# of elements in $W$. Considered on $L^{2}(B)$ it is the orthogonal projection onto the subspace $L^{2}(B)^{A l t}$ of anti-invariant elements; (this is clear since it is idempotent and self-adjoint and leaves fixed all anti-invariant $f$ 's). Alt also preserves each $\mathcal{H}_{l}$ by (7). Therefore it also preserves each $L^{2}(B)_{l, m}$, the subspace of $L^{2}(B)$ spanned by the functions (5) for fixed $l, m$. We denote the images of $A l t$ in these spaces by $L^{2}(B)_{l, m}^{A l t}$ and $\mathcal{H}_{l}^{\text {Alt }}$. We know that: $L^{2}(B)=\bigoplus_{l=0}^{\infty} \bigoplus_{m=1}^{\infty} L^{2}(B)_{l, m}$. It follows therefore that: $L^{2}(B)_{l, m}^{A l t}=\bigoplus_{l=0}^{\infty} \bigoplus_{m=1}^{\infty} L^{2}(B)_{l, m}^{A l t}$.

We claim that the restrictions to $\Omega$ of the functions in $L^{2}(B)_{l, m}^{A l t}(l=0,1, \ldots$; $m=1,2, \ldots$ ) span $L^{2}(\Omega)$ (and are still orthogonal to each other in $L^{2}(\Omega)$ for $l, m$ different). For this it is now enough to see that the restriction map (from $B$ to $\Omega$ ) is (up to a scalar) a Hilbert space isomorphism of $L^{2}(B)^{A l t}$ onto $L^{2}(\Omega)$. For this we note that (by the simple transitivity of $W$ on the chambers)

$$
\int_{B} f g=\sum_{w \in W} \int_{w \Omega} f g=\sum_{w \in W} \int_{\Omega} f\left(w^{-1} x\right) g\left(w^{-1} x\right) \mathrm{d} x=|W| \int_{\Omega} f(x) g(x) \mathrm{d} x,
$$

and that the restriction map is surjective, since for any $\varphi$ in $L^{2}(\Omega)$ we have the antiinvariant extension $\tilde{\varphi}$ on $L^{2}(B)$ defined uniquely by $\tilde{\varphi}(w x)=(\operatorname{det} w) \varphi(x)$ for $w \in W$ and $x \in \Omega$. We now have a complete description of the Dirichlet eigenfunctions of $\Omega$; they are given by (5) with the restriction that $h=h_{l} \in \mathcal{H}_{l}^{\text {Alt }}$.

We now only have to find the smallest $l$ such that $\mathcal{H}_{l}^{\text {Alt }} \neq 0$ (and a function in this $\mathcal{H}_{l}^{\text {Alt }}$ ) and to determine the constant $c_{\Omega}$.

By Bourbaki, [6] Ch. 5, $\S 5$, Prop. 5 (p. 113), П, defined in (8), is the lowest degree anti-invariant (with respect to $W$ ) polynomial on $\mathbb{R}^{n}$. Here is a possibly simpler proof of this. To show $\Pi$ is anti-invariant we have to show $\Pi \circ s_{H_{0}}=-\Pi\left(\forall H_{0} \in \mathcal{Z}\right)$. Now

$$
\Pi\left(s_{H_{0}} x\right)=\prod_{H \in \mathcal{Z}}\left(s_{H_{0}} x \mid e_{H}\right)=\prod_{H \in \mathcal{Z}}\left(x \mid s_{H_{0}} e_{H}\right) .
$$

As $H$ runs through all hyperplanes in $\mathcal{Z}, s_{H_{0}} e_{H}$ will run through all $e_{H}$ with possible sign changes. It follows that $\Pi \circ s_{H_{0}}= \pm \Pi$. To determine the sign it suffices to look 
at one special $x \in \mathbb{R}^{n}$. We take an $x$ that is very close to the plane $H_{0}$ and far from all other planes in $\mathcal{Z}\left(\left(x \mid e_{H_{0}}\right)\right.$ is small; $\left(x \mid e_{H}\right)$ is large for $\left.H \neq H_{0}\right)$. Then $s_{H_{0}} x$ is close to $x$, so $\left(s_{H_{0}} x \mid e_{H}\right)$ for $H \neq H_{0}$ has the same sign as $\left(x \mid e_{H}\right)$ but $\left(s_{H_{0}} x \mid e_{H_{0}}\right)=\left(x \mid s_{H_{0}} e_{H_{0}}\right)=$ $-\left(x \mid e_{H_{0}}\right)$. So $\Pi\left(s_{H_{0}} x\right)=-\Pi(x)$, and $\Pi \circ s_{H_{0}}=-\Pi$.

To show that $\Pi$ is of lowest degree, following Bourbaki [6], we observe that any semi-invariant is 0 on every $H \in \mathcal{Z}$, hence divisible by $\left(x \mid e_{H}\right)$, hence divisible by $\Pi$.

In addition, it is well known that $\Pi$ is harmonic. This can be seen as follows. Since Alt commutes with $\Delta$ and $\Pi$ is anti-invariant, $\Delta \Pi=\Delta \operatorname{Alt}(\Pi)=\operatorname{Alt}(\Delta \Pi)$, so $\Delta \Pi$ is anti-invariant. But the degree of $\Delta \Pi$ is strictly less than $\operatorname{deg}(\Pi)$, hence $\Delta \Pi=0$.

Now from (5) and from the inequalities for the $k_{v, m}$ we see that the principal eigenfunction is given by

$$
|x|^{-v} J_{v}\left(|x| k_{\nu, 1}\right) \Pi(x) \text {. }
$$

Once the principal eigenfunction has been found it suffices to use Theorem 1.1 to determine the asymptotically least favorable prior density on $\Omega$ given by

$$
g_{\Omega}(x)=c_{\Omega}|x|^{-2 v}\left(J_{v}\left(|x| k_{v, 1}\right)\right)^{2}(\Pi(x))^{2} .
$$

In order to determine the constant $c_{\Omega}$, since $\int g_{\Omega}(x) \mathrm{d} x=1$, we have, using polar coordinates, $x=r x^{\prime}, x \in \Sigma$ with $\Sigma$ denoting the unit sphere.

$$
\begin{aligned}
\frac{1}{c_{\Omega}} & =\int_{\Omega}\left(|x|^{-v} J_{v}\left(|x| k_{v, 1}\right) \Pi(x)\right)^{2} \mathrm{~d} x \\
& =\frac{1}{|W|} \int_{0}^{1} r\left(J_{v}\left(k_{v, 1} r\right)\right)^{2} \mathrm{~d} r \int_{\Sigma}\left(\Pi\left(x^{\prime}\right)\right)^{2} \mathrm{~d} \sigma\left(x^{\prime}\right) .
\end{aligned}
$$

By Watson [30, Section 18.1], the first integral on the right hand side equals $\frac{1}{2}\left(J_{v+1}\left(k_{v, 1}\right)\right)^{2}$. We denote the second integral by $K_{\Omega}$ and we consider

$$
L_{\Omega}=\int_{\mathbb{R}^{n}} \mathrm{e}^{-|x|^{2} / 2}(\Pi(x))^{2} \mathrm{~d} x .
$$

Using polar coordinates again, we obtain

$$
L_{\Omega}=2^{v} \Gamma(v+1) K_{\Omega} .
$$

On the other hand, Macdonald [24] pointed out that $\Pi$ being a harmonic polynomial, the integral $L_{\Omega}$ is equal to the norm computed by R. Steinberg and written down in the Appendix of Harder [15] namely

$$
L_{\Omega}=(2 \pi)^{n / 2} \frac{|W|}{2^{N}} \prod_{l=1}^{n}\left(m_{l} !\right)
$$

where $m_{1}, \ldots, m_{n}$ are the exponents of $W$. 
Thus,

$$
\frac{1}{c_{\Omega}}=\frac{\pi^{n / 2}}{2^{2 N} \Gamma\left(N+\frac{n}{2}\right)}\left(J_{v+1}\left(k_{v, 1}\right)\right)^{2} \prod_{l=1}^{n}\left(m_{l} !\right)
$$

with $N+\frac{n}{2}=v+1$.

Remark. - The exponents $m_{l}$ are given in Bourbaki [6] for $W$ arising from a root system in the tables on pp. 250-276 and for $W$ of type $H_{3}$ or $H_{4}$ on pp. 231-232. In the only remaining case, $W$ of type $I_{2}(p)$, we have trivially $m_{1}=1, m_{2}=p-1$.

Let us now consider the special domains given in (4). For an $n$-vector $x=\left(x_{i}, \ldots, x_{n}\right)$ and a positive $t$ let us introduce the following notation:

$$
x_{t}=\left(x_{1, t}, x_{2, t}, \ldots, x_{n, t}\right)
$$

where $x_{i, t}=\frac{x_{i}}{t}(1 \leqslant i \leqslant n)$. Note that if $x_{t} \in \Omega$ then $x \in t \Omega$.

COROLLARY 2.1. - The asymptotically least favorable prior densities for $t \mathcal{Q}, t \mathcal{A}$ and $t \mathcal{B}$ where $\mathcal{Q}, \mathcal{A}$ and $\mathcal{B}$ are defined in (4) are given respectively by

$$
\begin{aligned}
g_{t \mathcal{Q}}\left(x_{1}\right) & =c_{\mathcal{Q}} t^{-n}\left[\left|x_{t}\right|^{-\frac{3 n-2}{2}} J_{\frac{3 n-2}{2}}\left(k_{\frac{3 n-2}{2}, 1}\left|x_{t}\right|\right) x_{1, t} \cdots x_{n, t}\right]^{2}, \\
g_{t \mathcal{A}}(x) & =2^{-\frac{n(n-1)}{2}} c_{\mathcal{A}} t^{-n}\left[\left|x_{t}\right|^{-\frac{n^{2}-2}{2}} J_{\frac{n^{2}-2}{2}}\left(k_{\frac{n^{2}-2}{2}, 1}\left|x_{t}\right|\right) \prod_{i<j}\left(x_{i, t}-x_{j, t}\right)\right]^{2}, \\
g_{t \mathcal{B}}(x) & =2^{-n(n-1)} c_{\mathcal{B}} t^{-n}\left[\left|x_{t}\right|^{-\frac{n-2}{2}} J_{\frac{2 n^{2}+n-2}{2}}\left(k_{\frac{2 n^{2}+n-2}{2}, 1}\left|x_{t}\right|\right) \prod_{i=1}^{n} x_{i, t} \prod_{i<j}\left(x_{i, t}-x_{j, t}\right)^{2}\right]^{2} .
\end{aligned}
$$

Proof. - By Theorem 1.1 it suffices to use Theorem 2.2 and to determine the principal eigenfunction for each domain.

The case of $\mathcal{A}$, for example, is the special case where $\mathcal{Z}$ is the set of all hyperplanes $x_{i}-x_{j}=0,(i>j)$. Here $N=|\mathcal{Z}|=n(n-1) / 2$, and $\Pi(x)=2^{-N / 2} \prod_{i<j}\left(x_{i}-x_{j}\right)$. (So $v=\left(n^{2}-2\right) / 2$.)

The case where $\mathcal{B}=\left\{x \in \mathbb{R}^{n} ;|x| \leqslant 1\right.$ and $\left.x_{1} \geqslant x_{2} \geqslant \cdots \geqslant x_{n}>0\right\}$ corresponds to $\mathcal{Z}$, the set of all $x_{i}-x_{j}=0, x_{i}+x_{j}=0,(i>j), x_{i}=0$. So $N=|\mathcal{Z}|=n^{2}$, and $\Pi(x)=2^{-n(n-1) / 2} \prod_{i=1}^{n} x_{i} \prod_{i<j}\left(x_{i}^{2}-x_{j}^{2}\right)$.

Analogous arguments work for $\mathcal{Q}$ and $\mathcal{S}$. The explicit values of the constants $c_{\mathcal{S}}$, $c_{\mathcal{Q}}, c_{\mathcal{A}}$ and $c_{\mathcal{B}}$ can be obtained in a similar fashion by specifying the parameters in the formula for $c_{\Omega}$ given in Theorem 2.2.

Remark. - Chapter VI, $\S 4$ of Bourbaki [6] contains a list of all possible $W$ 's (and $C$ 's). For each case we can write down explicit formulas for $g_{t \Omega}(x)$ where $\Omega=C \cap B$.

\section{Construction of asymptotically minimax estimators}

As in Section 2, we consider domains $\Omega=C \cap B$ where $C$ is the fundamental chamber for a finite group generated by reflections and $B$ is the unit ball in $\mathbb{R}^{n}$. We denote by $\lambda$ 
the smallest eigenvalue of the Dirichlet eigenvalue problem (2) for $\Omega$. Our goal is to construct a family of estimators $\delta_{t}^{*}(x)$ associated to the domains $t \Omega(t>0)$ which is asymptotically minimax in the sense that

$$
\sup _{\omega \in t \Omega}\left|R\left(\omega, \delta_{t}^{*}\right)-\left(n-\frac{4 \lambda}{t^{2}}\right)\right|=\mathrm{o}\left(\frac{1}{t^{2}}\right) .
$$

The essential idea of the proof that an estimator $\delta_{t}^{*}$ satisfies (11) involves expressing the estimator as

$$
\delta_{t}^{*}(x)=x-\psi^{(t)}(x)
$$

and substituting this expression in Stein's unbiased estimate of the risk:

$$
R\left(\omega, \delta_{t}^{*}\right)=n-\int_{\mathbb{R}^{n}}\left(2 \operatorname{div} \psi^{(t)}(x)-\left|\psi^{(t)}(\omega)\right|^{2}\right) \phi_{n}(x-\omega) \mathrm{d} x,
$$

where div denotes divergence, $\left|\psi^{(t)}(\omega)\right|$ denotes the norm in $\mathbb{R}^{n}$ and $\phi_{n}(x)$, the standard normal $n$-dimensional density function.

Clearly the Bayes estimator

$$
\delta_{t}(x)=x+\operatorname{grad} \log \left(g_{t} * \phi_{n}\right)
$$

associated to the "approximately least favorable density" $g_{t}$ defined in Theorem 1.1 and explicitly computed in Theorem 2.2 does not have the property defined by (11), because as noted by Bickel [5] and Berkin and Levit [4], since $v_{\Omega}$ vanishes on the boundary of $\Omega$, the behaviour of $\nabla v_{\Omega} / v_{\Omega}$ is unstable near the boundary. This renders it necessary to construct $\delta_{t}^{*}$, a suitable modification of $\delta_{t}$, by considering an appropriate neighbourhood $\Omega^{(t)}$ of $\Omega$ and analogously to Bickel's work [5] in the one dimensional case, modifying $v_{\Omega}(x)$ from Theorem 1.1 inside and outside $\Omega^{(t)}$ in such a way that we can prove that the following error terms are uniformly small:

$$
\begin{aligned}
& R_{1 t}=\max _{\omega \in t \Omega}\left|\int_{t \Omega^{(t)}}\left(2 \operatorname{div} \psi^{(t)}(x)-\left\|\psi^{(t)}(x)\right\|^{2}-\frac{4 \lambda}{t^{2}}\right) \phi_{n}(x-\omega) \mathrm{d} x\right|=\mathrm{o}\left(\frac{1}{t^{2}}\right), \\
& R_{2 t}=\max _{\omega \in t \Omega}\left|\int_{\left(t \Omega^{(t)}\right) \mathcal{C}}\left(2 \operatorname{div} \psi^{(t)}(x)-\left\|\psi^{(t)}(x)\right\|^{2}-\frac{4 \lambda}{t^{2}}\right) \phi_{n}(x-\omega) \mathrm{d} x\right|=\mathrm{o}\left(\frac{1}{t^{2}}\right) .
\end{aligned}
$$

We proceed to construct $\Omega^{(t)}$ and $\delta_{t}^{*}$ as follows. In order to simplify notation, let $e_{i}(1 \leqslant i \leqslant N)$ denote the vectors $e_{H}$ of $(8)$ arranged in some order. Let us choose a unit vector $u \in C$ so that $\left(u \mid e_{i}\right) \geqslant 0 \forall i$ and let us choose $k$ such that $k \geqslant 1 / \min _{i}\left(u \mid e_{i}\right)$. Let $a_{t}<1$ satisfying $a_{t} \downarrow 0$ and $t a_{t} \rightarrow \infty$. Now let $b_{t}$ be such that $0<b_{t}<a_{t} /(2(1+(k+$ 1) $\left.\left.a_{t}\right)\right)$ and $b_{t}^{-4} \int_{|x|>\frac{t a t}{2}} \phi_{n}(x) \mathrm{d} x \rightarrow 0$.

Let $|x|=r$ and let

$$
\begin{aligned}
\Omega & =\{x \in C:|x| \leqslant 1\}, \\
\Omega_{t} & =\left\{x \in C: r<1-b_{t} \text { and }\left(x \mid e_{i}\right)>b_{t}(1 \leqslant i \leqslant N)\right\}, \\
\Omega^{(t)} & =\left(1+(k+1) a_{t}\right) \Omega_{t}-a_{t} k u .
\end{aligned}
$$


It is easy to see that, if $\omega \in \Omega$ and $x \in\left(\Omega^{(t)}\right)^{\mathcal{C}}$, then $|x-\omega|>\frac{a_{t}}{2}$.

We define $\bar{\psi}$, for $x \in \Omega$ only, by

$$
\bar{\psi}(x)=\frac{-\nabla g_{\Omega}(x)}{g_{\Omega}(x)},
$$

where $g_{\Omega}$ is as in Theorem 2.2. From $\Delta g_{\Omega}(x)=-\lambda g_{\Omega}(x)$ it follows that

$$
2 \operatorname{div} \bar{\psi}(x)-|\bar{\psi}(x)|^{2}=4 \lambda
$$

for all $x$ in $\Omega$.

Using the auxiliary functions

$$
\begin{array}{ll}
\varphi_{0}(y)=k_{v, 1} \frac{j_{v}^{\prime}\left(k_{v, 1} y\right)}{j_{v}\left(k_{v, 1} y\right)}, & (0 \leqslant y<1), \\
\varphi(y)=\frac{1}{y}, & (0<y),
\end{array}
$$

the explicit formula (9) gives

$$
\bar{\psi}(x)=-2 \varphi_{0}(r) \frac{x}{r}-2 \sum_{i} \varphi\left(\left(x \mid e_{i}\right)\right) e_{i} .
$$

We modify $\bar{\psi}(x)$ near the boundary of $\Omega$ and extend its definition to $\mathbb{R}^{n}$ by first setting

$$
\begin{aligned}
\varphi_{0, t}(y) & = \begin{cases}\varphi_{0}(y), & \text { if } 0 \leqslant y \leqslant 1-b_{t}, \\
\varphi_{0}\left(1-b_{t}\right)+\varphi_{0}^{\prime}\left(1-b_{t}\right)\left(y-\left(1-b_{t}\right)\right), & \text { if } y>1-b_{t},\end{cases} \\
\varphi_{t}(y) & = \begin{cases}\varphi(y)\left(=\frac{1}{y}\right), & \text { if } y \geqslant b_{t}, \\
\varphi\left(b_{t}\right)+\varphi^{\prime}\left(b_{t}\right)\left(y-b_{t}\right), & \text { if } y<b_{t},\end{cases}
\end{aligned}
$$

and then for $x \in \mathbb{R}^{n}$ and $r=|x|$

$$
\begin{aligned}
\psi_{0, t}(x) & =-2 \varphi_{0, t}(r) \frac{x}{r}, \\
\psi_{i, t}(x) & =-2 \varphi_{t}\left(\left(x \mid e_{i}\right)\right) e_{i}, \\
\psi_{t}(x) & =\psi_{0, t}(x)+\sum_{i} \psi_{i, t}(x) .
\end{aligned}
$$

Finally, we define,

$$
\psi^{(t)}(x)=\frac{1}{\left(1+(k+1) a_{t}\right) t} \psi_{t}\left(\frac{1}{\left(1+(k+1) a_{t}\right) t}\left(x+a_{t} k u\right)\right) .
$$

THEOREM 3.1. - An asymptotically minimax estimator $\delta_{t}^{*}(x)$ which satisfies (11) can be defined by letting

$$
\delta_{t}^{*}(x)=x-\psi^{(t)}(x),
$$

where $\psi^{(t)}$ is defined in (17). 
Proof. - We will use the following properties in the proof.

(a) $2 \operatorname{div} \psi^{(t)}(x)-\left\|\psi^{(t)}(x)\right\|^{2}=\frac{4 \lambda}{\left(1+(k+1) a_{t}\right)^{2} t^{2}}$ for $x \in t \Omega^{(t)}$. This follows from (12) and from the fact that $\psi_{t}=\bar{\psi}$ on $\Omega_{t}$.

(b) $2 \operatorname{div} \psi_{t}(x)-\left\|\psi_{t}(x)\right\|^{2} \leqslant \frac{c}{b_{t}^{4}}\left(r^{2}+1\right)$ for some $c$ independent of $x$ and $t$.

To see this, we first get by direct computation

$$
\begin{aligned}
\operatorname{div} \psi_{o t}(x) & =-2\left(\varphi_{o t}^{\prime}(r)+\varphi_{o t}(r) \frac{n-1}{r}\right), \\
\operatorname{div} \psi_{i t}(x) & =-2 \varphi_{t}^{\prime}\left(\left(x \mid e_{i}\right)\right), \\
\left|\psi_{o t}(x)\right|^{2} & =4 \varphi_{o t}(r)^{2}, \\
\left|\psi_{i t}(x)\right|^{2} & =4 \varphi_{t}\left(\left(x \mid e_{i}\right)\right)^{2} .
\end{aligned}
$$

Then we observe that the functions $\varphi_{t}^{\prime}(y), \varphi_{t}(y)$ (for $y \in \mathbb{R}$ ) and $\varphi_{o t}^{\prime}(y), \varphi_{o t}(y)$ (for $y \geqslant 0$ ) are all majorized by

$$
\frac{c}{b_{t}^{2}}(y+1)
$$

for some constant $c$. This is obvious for $\varphi_{t}^{\prime}$ and $\varphi_{t}$; to prove it for $\varphi_{o t}^{\prime}$ we note that, $k_{\nu, 1}$ being a simple zero and the smallest positive zero of $j_{\nu}$, we have, for $0<y<1$, $\varphi_{0}^{\prime}(y)=-\frac{1}{(1-y)^{2}}+a(y)$ for a smooth function $a(y)$ on $[0,1]$. From this the estimate for $\varphi_{o t}^{\prime}$ and then for $\varphi_{o t}$ follows and (b) is then an immediate consequence.

Now, to prove the theorem, we have to show that assertions (14) and (15) are true. It is easy to see that

$$
\left(\frac{4 \lambda}{\left(1+(k+1) a_{t}\right)^{2} t^{2}}-\frac{4 \lambda}{t^{2}}\right) \int_{t \Omega^{(t)}} \phi_{n}(x-\omega) \mathrm{d} x=\mathrm{o}\left(\frac{1}{t^{2}}\right),
$$

and this implies (14) by (a). So it is enough to prove that $R_{2 t}$ in (15) is uniformly small. In other words, we have to prove that

$$
\begin{aligned}
& \max _{\omega \in t \Omega} \mid \int_{\left(t \Omega^{(t)}\right)^{c}}\left\{\frac{1}{\left(1+(k+1) a_{t}\right)^{2}}\left(2 \operatorname{div} \psi_{t}-\left\|\psi_{t}\right\|^{2}\right)\right. \\
& \left.\quad \times\left(\frac{1}{\left(1+(k+1) a_{t}\right) t}\left(x+a_{t} k u\right)\right)-4 \lambda\right\} \phi_{n}(x-\omega) \mathrm{d} x \mid=\mathrm{o}(1) .
\end{aligned}
$$

As previously observed if $x \in\left(t \Omega_{(t)}\right)^{c}$ and $\omega \in t \Omega$, then $|x-\omega|>\frac{t a_{t}}{2}$. Changing the variable $z=x-\omega$, and applying (b), we find that the left hand side of (19) is majorized by

$$
\frac{c^{\prime \prime}}{b_{t}^{4}} \int_{|z|>\frac{t a t}{2}}\left(|z|^{2}+c\right) \phi_{n}(z) \mathrm{d} z
$$

and this is really o(1) by the hypotheses about $a_{t}$ and $b_{t}$. 


\section{Acknowledgements}

The first author was partially supported by NSF of the USA and by a PSC-CUNY award. The second author would like to acknowledge the partial support of NSERC of Canada and FCAR of Québec for this research. Both authors are grateful to the referees for suggestions and for indicating certain references.

\section{REFERENCES}

[1] Berard P., Spectres et groupes cristallographiques I: Domaines euclidiens, Inventiones Mathematicae 58 (1980) 179-199.

[2] Berard P., Besson G., Spectres et groupes cristallographiques II: Domaines spheriques, Annales de l'Institut Fourier 30 (1980) 237-248.

[3] Berger J.O., Statistical Decision Theory and Bayesian Analysis, 2nd ed., Springer-Verlag, New York, 1985.

[4] Berkin P.E., Levit B.Ya., Second-order asymptotically minimax estimates for the mean of a normal population, Problemy Peredachi Informatsii 16 (3) (1980) 60-79. Translation: Problems of Information Transmission (1981) 212-229.

[5] Bickel P.J., Minimax estimation of the mean of a normal distribution when the parameter space is restricted, Ann. Statist. 9 (1981) 1301-1309.

[6] Bourbaki N., Éléments de mathématique, Fascicule XXXIV, Groupes et algèbres de Lie, Chapitres IV, V et VI, Hermann, Paris, 1969.

[7] Brown L.D., Statistical Decision Theory, Mimeographed Notes, Cornell University, Ithaca, NY, 1979.

[8] Brown L.D., Gajek L., Information inequalities for the Bayes risk, Ann. Statist. 18 (1990) 1578-1594.

[9] Casella G., Strawderman W., Estimating a bounded normal mean, Ann. Statist. 9 (1981) 868-876.

[10] Donoho D.L., Liu R.C., MacGibbon B., Minimax risk over hyperrectangles and implications, Ann. Statist. 18 (1990) 1416-1437.

[11] Erdélyi A., The Bateman Manuscript Project, Higher Transcendental Functions (3 volumes), McGraw-Hill, New York, 1953-1955 (Erdélyi A. (Ed.)).

[12] Ferguson T.S., Mathematical Statistics, A Decision Theoretic Approach, Academic, New York, 1967.

[13] Folland G.B., Introduction to Partial Differential Equations, 2nd ed., Princeton University Press, 1995.

[14] Gilbarg D., Trudinger N.S., Elliptic Partial Differential Equations of Second Order, 2nd ed., Springer-Verlag, Berlin, 1983.

[15] Harder G., A Gauss-Bonnet formula for discrete arithmetically defined groups, Ann. Sci. Ec. Norm. Super. 4 (1971) 409-445.

[16] Johnstone I.M., MacGibbon K.B., Minimax estimation of a constrained Poisson vector, Ann. Statist. 20 (1992) 807-831.

[17] Johnstone I.M., MacGibbon K.B., Asymptotically minimax estimation of a constrained Poisson vector via polydisc transforms, Annales de l'Institut Henri Poincaré, Probabilité et Statistiques 29 (1993) 289-319.

[18] Kempthorne P.J., Numerical specification of discrete least favorable prior distributions, SIAM J. Sci. Statist. Comput. 8 (1987) 171-184. 
[19] Levit B.Ya., On asymptotic minimax estimates of the second order, Theor. Probab. Appl. 25 (1980) 552-568 (Translation).

[20] Levit B.Ya., Minimax estimation and positive solutions of elliptic equations, Theor. Probab. Appl. 27 (1982) 563-586 (Translation).

[21] Levit B.Ya., Second order asymptotic optimality and positive solutions of Schrödinger's equation, Theor. Probab. Appl. 30 (1985) 333-363 (Translation).

[22] Levit B.Ya., Evaluation of the minimax risk, in: Proc. Fourth International Vilnius Conf. Prob. Theory Math. Stat., Vilnius, Vol. 4, 1985, pp. 181-183.

[23] Levit B.Ya., On second order admissibility in simultaneous estimation, in: Prohorov Yu.A., Sazonov V.V. (Eds.), Proc. 1st. World Congress Bernoulli Soc. Tashkent, USSR, VNU Sc. Press, Ultrecht, 1986.

[24] MacDonald P.G., The volume of a compact Lie Group, Inventiones Mathematicae 56 (1980) 93-95.

[25] Robertson T., Wright F.T., Dykstra R.L., Order Restricted Statistical Inference, Wiley, New York, 1988.

[26] Schoenfeld D.A., Confidence bounds for normal means under order restrictions, with applications to dose-response curves, toxicology experiments, and low-dose extrapolation, J. Amer. Statist. Assoc. 81 (1986) 186-195.

[27] Urakawa H., Bounded domains which are isospectral but not congruent, Ann. Sci. Ec. Norm. Super. IV (15) (1982) 441-456.

[28] Urakawa H., Reflection groups and the eigenvalue problems of vibrating membranes with mixed boundary conditions, Tohoku Math. J. II (36) (1984) 175-183.

[29] Wald A., Statistical Decision Functions, Wiley, New York, 1950.

[30] Watson G., A Treatise on the Theory of Bessel Functions, Cambridge University Press, Cambridge, 1962.

[31] Zinzius E., Minimaxschatzer für den Mittelwert $v$ einer normalverteilten Zufallgröße mit bekannter Varianz bei vorgegebener oberer und unterer Schranke für $v$, Math. Operationsforsch. Statist. Ser. Statist. 12 (1981) 551-557. 